\title{
Seminare / Séminaires
}

\section{Praxiseröffnung/-übernahme}

\section{Themen}

Juristische Aspekte (Praxisbewilligung, Zulassung zur Sozialversicherung, Vertragswesen), Gesellschaftsformen / Ehe- und Erbrecht (Trennung Privat- vom Geschäftsvermögen, Ehegüterstand, Erbschaftsplanung), Praxiseinrichtung (Inneneinrichtung, Kostenberechnung), Praxisadministration (Leistungserfassungsund Abrechnungssysteme), Unternehmensbewertung einer Arztpraxis (Berechnung und Beurteilung des Unternehmenswertes), Finanzierung der Arztpraxis (Businessplan, Kredite, Absicherungsmöglichkeiten), Versicherungen/Vorsorge/Vermögen (Personen- und Sachversicherungen, Vorsorgeplanung).

\section{Sponsoren}

Die Kosten werden durch diverse Sponsoren (siehe www.fmhservices.ch) gedeckt.

\section{Daten}

К04

Donnerstag, 1. September 2011 9.00-16.30 Uhr

Zürich

FMT

K05

Donnerstag, 3. November 2011 9.00-16.30 Uhr

Basel Hotel Victoria

\section{Praxisübergabe}

Das Seminar richtet sich an zukünftige Praxisübergeber/innen. Hinweis: Vor allem aus steuer- und vorsorgeplanerischer Sicht lohnt es sich, sich bereits frühzeitig (5-10 Jahre) mit diesem Thema auseinanderzusetzen.

\section{Themen}

Juristische Aspekte (Praxisübergabevertrag, allg. Vertragswesen, Übergabe der Krankengeschichten), Unternehmensbewertung einer Arztpraxis (Berechnung Inventarwert und Goodwill als Verhandlungsbasis),

Annullierungsbedingungen / Conditions d'annulation Bei Abmeldungen oder Fernbleiben werden folgende Unkostenbeiträge erhoben:

Un montant est perçu pour une absence ou une annulation. Il est de:

- 50 CHF pro Person ab 14 Tage vor Seminarbeginn / par personne dans les 15 jours avant le début du séminaire;

- 100 CHF pro Person ab 7 Tage vor Seminarbeginn oder Fernbleiben / par personne dans les 7 jours avant le début du séminaire.
Versicherungen/Vorsorge/Vermögen (Übergabe/Auflösung von Versicherungsverträgen, Pensions- und Finanzplanung), Steuern (Steueraspekte bei der Praxisübergabe, Optimierung der steuerlichen Auswirkungen, Liquidations- und Grundstückgewinnsteuer, Bestimmung des optimalen Übergabezeitpunktes).

\section{Sponsoren}

Die Kosten werden durch diverse Sponsoren (siehe www.fmhservices.ch) gedeckt.

\section{Daten}

K09

Donnerstag, 8. September 2011 Zürich 13.30-18.00 Uhr FMT

K10 Donnerstag, 10. November 2011 Basel 13.30-18.00 Uhr

\section{Finanz- und Steuerplanung}

\section{Themen}

Finanzplanung (Businessplan, Buchhalterische Massnahmen vor Praxiseröffnung/-übernahme, Standardkontenplan für Ärzte, System der doppelten Buchhaltung, EDV-unterstützte Buchführungslösung), Steuern (Steueraspekte bei Eintritt in die Selbständigkeit, Steuerfallen und Steuerrisiken, optimierte Steuerplanung).

\section{Sponsoren}

Die Kosten werden durch diverse Sponsoren (siehe www.fmhservices.ch) gedeckt.

\section{Datum}

K12 Donnerstag, 15. September 2011 Bern 13.30-18.00 Uhr Schmiedstube

\section{Ouverture et reprise d'un cabinet médical}

\section{Contenu}

Business plan (préparation du plan de financement et crédit d'exploitation, financement par la banque), Aménagement (implantation, projet et concept d'aménagement, choix du mobilier, budget), Estimation d'un cabinet (inventaire et goodwill), Administration d'un cabinet médical (dans le cabinet, par la banque), Assurances (toutes les assurances à l'intérieur et autour du cabinet), Passage du statut de salarié à celui d'indépendant et fiscalité.

\section{Sponsors}

Les coûts sont pris en charge par divers sponsors (voir www.fmhservices.ch).

\section{Dates}

\begin{tabular}{|lll} 
K22 & $\begin{array}{l}\text { Jeudi, } 1 \text { septembre } 2011 \\
17.00-21.30 \mathrm{~h}\end{array}$ & $\begin{array}{l}\text { Lausanne } \\
\text { World Trade Center }\end{array}$ \\
K23 & $\begin{array}{l}\text { Jeudi, } 10 \text { novembre } 2011 \\
17.00-21.30 \mathrm{~h}\end{array}$ & $\begin{array}{l}\text { Genève } \\
\text { Ramada Park Hôtel }\end{array}$
\end{tabular}

\section{Anmeldung und Auskunft / Inscription et information}

www.fmhservices.ch oder FMH Consulting Services, Cornelia Steinmann, Burghöhe 1, 6208 Oberkirch, Tel. 04192500 77, Fax 0419210586.

\section{Hinweis / Remarque}

Bei sämtlichen Seminaren, bei denen die Kosten teilweise oder gänzlich von Seminarsponsoren gedeckt werden, werden die Teilnehmeradressen den jeweiligen Sponsoren zur Verfügung gestellt.

Les adresses des participants aux séminaires dont les coûts sont couverts en partie ou totalement par des sponsors sont communiquées aux sponsors concernés. 